\title{
Sentido de comunidad, participación y apego de lugar en comunidades desplazadas y no desplazadas post desastres: Chaitén y Constitución*
}

\author{
Sense of Community, Participation and Place \\ Attachment in Post Disasters Displaced and Non- \\ displaced Communities: Chaitén and Constitución
}

Enviado: 1ํ de marzo de 2015 | Revisado: 1ํ de junio de 2015 | Aceptado: $1^{\text {o }}$ de agosto de 2015

\author{
HÉCTOR BERROETA ** \\ ÁlVARO RAMONEDA \\ LUIS OPAZO \\ Escuela de Psicología, Universidad de Valparaíso, Chile
}

\section{doi:10.11144/Javeriana.up14-4.scpa}

Para citar este artículo: Berroeta, H., Ramoneda, Á., \& Opazo, L. (2015) Sentido de comunidad, participación y apego de lugar en comunidades desplazadas y no desplazadas post desastres: Chaitén y Constitución. Universitas Psychologica, 14(4), 1221-1234. http://dx.doi.org/10.11144/Javeriana. up14-4.scpa

* Artículo de investigación. Este trabajo fue financiado por el proyecto FONDECYT 11121596 "Vínculos socio-espaciales en contextos de transformación urbana producida por catástrofes naturales".

** hector.berroeta@uv.cl

\section{RES UMEN}

La relación socio-espacial de personas y comunidades se ve afectada por procesos de desplazamientos a causa de desastres socio-naturales. A partir de experiencias de la comunidad disgregada de Chaitén por la erupción del volcán Chaitén y la que se mantuvo unida en Constitución después del terremoto de 2010, se exploró cómo los procesos vividos inciden en niveles de apego e identidad de lugar, así como sentido de comunidad y participación. Las comunidades estudiadas presentan diferentes vínculos espaciales y relacionales con respecto a su barrio de origen y actual. Las diferencias presentadas evidencian que vínculos comunitarios como aspectos psicológicos deben ser considerados centrales en la elaboración e implementación de estrategias para desplazamientos o reconstrucción. Se encuestó a un total de 224 personas.

Palabras clave

sentido de comunidad; apego de lugar; identidad de lugar; desastres; participación; desplazamiento

\section{A B S T R A C T}

Socio spatial relationships of people and communities are affected by processes of displacement due to socio-natural disasters. Based on the experiences of the disintegrated community of Chaitén by the eruption of the Chaitén volcano and the community which remained united in Constitution after the earthquake of 2010, this research explores how the experienced processes affect levels of place identity and place attachment, as well as in sense of community and participation. The studied communities express different spatial and relational links with their origin and actual neighborhood. Differences evidence that community bonds and psychological aspects must be considered as central in the development and implementation of displacement or reconstruction strategies post disasters. The survey considered a total of 224 individuals.

Keywords

sense of community; place attachment; place identity; disasters; participation; displacement 
El concepto de desastre ha transitado de natural a socio-natural. De acuerdo con Razeto (2013), es fundamental considerar la relación entre naturaleza y sociedad para ampliar la comprensión del fenómeno y las potenciales acciones de respuesta. Los desastres, al generar acciones colectivas, se constituyen no solo en catalizadores sociales, sino también en fenómenos sociales que obligan a las instituciones a pensar respuestas más allá de lo material.

Los estudios sobre desplazamiento involuntario establecen que el ajuste post-desplazamiento dependerá de las diferencias de calidad entre los viejos y los nuevos entornos residenciales, de las oportunidades para relacionarse, satisfacer necesidades de autoestima y sistemas de control y cambio comunitario (Heller, 1982). En estos contextos, como señala Manzo (2014), la tradicional manera de entender los vínculos con el lugar, se despliega de un modo complejo y multifacético que requiere de una reflexión sobre las condiciones en las que viven las personas afectadas por un desplazamiento.

Desde una perspectiva ambiental comunitaria, proponemos la necesidad de analizar la disrupción ambiental desde un marco centrado en la comunidad, que incorpore conceptos físicos y psicológicos (Manzo \& Perkins, 2006; Vidal, Berroeta, Di Masso, Valera, \& Pero, 2013) que aborden los vínculos con el lugar y con las personas (Mihaylov $\&$ Perkins, 2014).

Asumiendo esta perspectiva, en esta investigación se exploran las dimensiones afectivas (apego de lugar) y cognitivas (identidad de lugar) con el espacio y las dimensiones relacionales (sentido de comunidad) y conductuales (participación) con la vecindad, en dos comunidades afectadas por un desastre socio-natural: un desplazamiento debido a la erupción de un volcán y otro debido a un proceso de reconstrucción en el mismo asentamiento, a consecuencia de un terremoto. Específicamente planteamos la pregunta: iqué niveles de apego de lugar, identidad de lugar, sentido de comunidad y participación cívica alcanzan personas que fueron desplazadas tras una erupción volcánica y personas a las que se les reconstruyeron sus viviendas en el mismo lugar tras un terremoto? La hipótesis que subyace a esta investigación es que las personas que se mantuvieron en su lugar de origen presentan diferentes grados de apego de lugar, identidad de lugar, sentido de comunidad y participación de quienes fueron desplazados.

La literatura psicosocial, se ha centrado fundamentalmente en el estudio de las consecuencias psicológicas para los habitantes de las localidades afectadas por desastres (Bonanno, Galea, Bucciarelli, \& Vlahow, 2006, García, Reyes, \& Cova, 2014), dejando de lado el estudio psico-ambiental de estos fenómenos. Se ha establecido que la severidad de la reacción psicológica se relaciona negativamente con el bienestar (García et al., 2014), que dependerá de la magnitud de la destrucción y de las características demográficas y sociales de los habitantes (Norris, Friedman, \& Watson, 2002). Investigaciones realizadas tras eventos de terremotos y tsunamis concluyen que la pérdida del hogar es un factor de estrés primario mucho mayor que los procesos de desplazamiento. Joh (1997) reporta que aquellas personas que han perdido sus casas, sufren más estrés que aquellos que solo necesitan repararlas, a la vez que muestran mayores deseos de volver a vivir en los lugares devastados (Sanders, Browie, \& Bowie, 2003).

Desde una perspectiva psico-ambiental, diversos estudios plantean que el sentido de comunidad, el apego de lugar y la participación cívica son centrales en el grado de resiliencia que alcanzan las comunidades enfrentadas a desastres socio-naturales (Bird, Gísladóttir, \& Dominey-Howes, 2011; Norris, Stevens, Pfefferbaum, Wyche \& Pfefferbaum, 2008). El sentido de comunidad influye en el ajuste psicológico de la experiencia (Banyard \& Williams, 2007; Greenfield \& Marks, 2010) y los niveles de apego influyen en la aceptación del cambio ambiental (Ruiz \& Hernández, 2014).

Se sabe que el impacto de un desplazamiento post catástrofe es adverso (Norris, Watson, Hamblen, \& Pfefferbaum, 2005), sin embargo, se desconoce qué ocurre en comunidades que reconstruyen sus viviendas en el mismo lugar. No se cuenta con estudios que describan los vínculos espaciales (apego de lugar e identidad de lugar) y sociales (sentido de comunidad y participación) en comunidades afectadas por desastres socio-naturales, aún cuando se cuenta 
con amplia evidencia respecto a la importancia de estos factores en la calidad de vida comunitaria.

En estudios realizados por Perkins (Manzo \& Perkins, 2006; Perkins \& Long, 2002), se concluye que el apego de lugar y el sentido de comunidad desempeñan un papel importante en la revitalización de los vecindarios. Más concretamente, se afirma que en los casos en donde no hay conexión emocional de las personas a los lugares, estas no suelen estar lo suficientemente comprometidas para trabajar con los vecinos e instituciones locales en el mejoramiento de su entorno.

Existe un acuerdo en que el sentido de comunidad (SC) es la percepción de pertenencia, el sentimiento de cohesión y conexión emocional entre las personas de una comunidad que se basa en una historia e intereses compartidos (Long \& Perkins, 2003). El SC principalmente refiere a una experiencia de evaluación individual o colectiva que opera como un recurso de la vida en comunidad (Nowel \& Boyd, 2010). El SC se ha asociado al capital social (Norris et al.,, 2008), al bienestar psicológico y al compromiso comunitario (Nowel \& Boyd, 2010).

El planteamiento central de los principales estudios sobre identidad de lugar (Bonaiuto \& Bonnes, 2000; Proshansky, Fabian, \& Kaminoff, 1983; Twigger-Ross, Bonaiuto, \& Breakwell, 2003) es que la identidad personal se construye en relación al entorno físico del individuo. Igualmente, su identidad social se construye por su pertenencia a categorías sociales como: sexo, raza o clase social. Se considera entonces que la identidad de lugar es una dimensión del self, que define la identidad en relación con el entorno físico que satisface las necesidades biológicas, psicológicas, sociales y culturales (Proshansky et al., 1983).

El apego de lugar refiere al vínculo emocional que las personas establecen con los lugares (Lewicka, 2011; Scannell \& Gifford, 2010a; 2010b), sin importar si es positivo (Hidalgo \& Hernandez, 2001) o negativo (Fried, 1963). Una disrupción del apego de lugar afecta la auto definición individual y comunitaria (Brown \& Perkins, 1992) y puede dificultar la resiliencia comunitaria (Norris et al., 2008).

Laparticipación cívica incluye la participación en las actividades del barrio y se operativiza a través de la pertenencia a entidades o asistiendo a actividades (Perkins, Florin, Rich, Wandersman, \& Chavis, 1990). La participación en los asuntos del barrio se relaciona con el apego al barrio (Cuba \& Hummon, 1993). La participación es un ejemplo de la dimensión acción-transformación que, junto a la identificación simbólica, explican la apropiación del barrio (Korosek-Serfaty, 1976; Pol, 2002) y el apego al mismo (Vidal \& Pol, 2005). Recientemente Vidal et al (2013), prueban un modelo de ecuaciones estructurales que confirma la incidencia de la participación cívica en el sentido de comunidad y el apego de lugar, utilizando los mismos instrumentos que utilizamos en esta investigación, en cuatro barrios en Chile.

Considerando estos antecedentes y la escaza investigación en contextos de reasentamiento post desastres, en esta investigación optamos por una aproximación mixta poli-etápica, de aplicación de instrumentos cuantitativos y entrevistas grupales. En este texto se reportan los resultados de la primera fase cuantitativa.

\section{Dos casos, dos procesos}

Los casos estudiados corresponden a las ciudades de Chaitén y Constitución. Ambas localidades y sus habitantes vivieron procesos distintos post catástrofe en relación a la actuación del Estado en la resolución de las repercusiones negativas de los mismos. La ciudad de Chaitén se ubica en la décima región del país y está situada entre el mar y la cordillera. En ella se destacan tres volcanes principales, entre ellos el volcán Chaitén. El 2 de mayo de 2008 y como resultado de la erupción de este volcán, la ciudad tuvo que ser evacuada en un radio de 50 kilómetros. 4.700 personas correspondientes a 1.500 familias fueron ubicadas en ciudades como Puerto Montt y Futaleufú, entre otras (Marchant, 2010). A la fecha, los organismos públicos no tienen claridad sobre el destino de la comunidad residente fuera de Chaitén, ni sobre cómo se realizó cada uno de los procesos para la adquisición de sus nuevas viviendas.

El proceso posterior de adquisición de las nuevas viviendas se realizó de manera individual mediante 
un subsidio en dinero entregado por el Estado, con el que cada familia compró una nueva vivienda. Con este bono las familias gestionaron sus viviendas en el mercado inmobiliario (comunicación personal, Inostroza \& Millaquen, 2013). La comunidad quedó fragmentada porque no se generaron condiciones para una propuesta colectiva.

Producto de las indagaciones realizadas para esta investigación, se constató que en la actualidad aproximadamente 200 familias de chaiteninos viven en un amplio sector residencial ubicado al norte de la ciudad de Puerto Montt llamado Alerce. Aquí, se encuestó a familias provenientes de cuatro barrios distintos que residen en viviendas unifamiliares de alrededor de $44 \mathrm{mts}^{2}$ (comunicación personal, Inostroza \& Millaquen, 2013).

Diferente es el caso de estudio en Constitución, una ciudad costera ubicada en la séptima región del país. Producto del terremoto y posterior tsunami que impactó las costas chilenas el 27 de febrero de 2010 (Larrañaga \& Herrera, 2010) el conjunto habitacional Cerro O`Higgins resultó con daños irreparables en su infraestructura, razón por la que se determinó la demolición completa del condominio social. Tras un proceso de reconstrucción, en marzo de 2013 se entregó un nuevo condominio conformado por tres bloques de cuatro pisos y 48 departamentos, en el que actualmente residen 142 habitantes, todos pertenecientes a la comunidad de origen del barrio.

\section{Método}

\section{Participantes}

Tanto en Chaitén como en Constitución se realizó una encuesta a partir de una muestra no probabilística por conveniencia. Esta muestra está compuesta por 144 habitantes desplazados de la ciudad de Chaitén ( $\mathrm{N}=144), 95$ mujeres, 49 hombres, que se estima corresponden a casi la totalidad del universo de personas desplazadas que se encuentran en el sector ${ }^{1}$. Presentaron un promedio de edad de 45.12

1 No existen estadísticas oficiales con respecto a cuántos desplazados de Chaitén se encuentran en Puerto Montt. Se indagó en la Intendencia del lugar, pero ellos tampoco tienen información años $(D E=16.976)$ y una media de años viviendo en el barrio actual de 3.48 años $(D E=1.540)$. En Constitución participaron 80 personas ( 22 mujeres, 58 hombres) de un universo de 142 habitantes que presentaron un promedio de edad de 42.71 años $(D E=14.664)$ y una media de años viviendo en el barrio actual de 12.42 años ( $D E=7.061)$. El total de participantes perdió su vivienda a consecuencia de los desastres.

\section{Técnicas}

Para medir apego social y espacial, se adaptaron las escalas de Scannell y Gifford (2010b), que en su versión original reportan un Alfa de Cronbach de 0.78 , que se componen de cuatro y cinco ítems respectivamente, con formato de respuesta tipo Likert $(1=$ Nada a 6 = Muchísimo), que se respondían con relación al barrio de origen y al actual. Por ejemplo: "En ese lugar vivía gente como yo" y "Aquí los espacios públicos son especiales". Se realizó un análisis factorial exploratorio que corroboró la estructura factorial de las escalas originales. La confiabilidad de la escala medida mediante el coeficiente Alpha de Cronbach es, para apego social pasado (PASBp) 0.914; para apego espacial pasado (PAEBp) 0.884; apego social actual (PASBa) 0.898; y apego espacial actual (PAEBa) 0.885. El puntaje individual en la escala se obtuvo calculando el promedio de los ítems.

Para medir el grado de identificación con el lugar se adaptó la escala global de identidad de lugar de Vidal, Valera y Peró (2010), tomada de Hernández, Hidalgo, Salazar y Hess (2007) cuyo Alfa de Cronbach es 0.94. La escala se compone de cinco ítems con formato de respuesta tipo Likert que debían ser respondidos respecto al barrio de origen, al actual y a la ciudad. Por ejemplo: "Siento que pertenezco a este barrio". Se realizó un análisis factorial exploratorio que corroboró la estructura factorial de la escala original. La confiabilidad de la escala, medida con el coeficiente Alpha de Cronbach para identidad de lugar pasado (PIBp) es 0.920 y para identidad de lugar actual (PIBa) es 0.892 .

\footnotetext{
al respecto, por lo que los datos surgen a partir de la investigación
} en terreno. 
Para la medida sentido de comunidad se adaptó la escala breve de Long y Perkins (2003), que en su versión original reporta un Alfa de Cronbach de 0.74. Las escala se compone de ocho ítems con formato de respuesta tipo Likert, que debían responderse con relación al barrio actual. Por ejemplo: "Muchos de mis vecinos me conocen". Se realizó un análisis factorial exploratorio que corroboró la estructura original de la escala. La confiabilidad de la escala, medida mediante el coeficiente Alpha de Cronbach es de 0.838. El puntaje individual en la escala se obtuvo calculando el promedio de los ocho ítems.

Para la medida de participación cívica se adaptó la escala de Long y Perkins (2003) que en su versión original reporta un Alfa de Cronbach de 0.78. La escala se compone de diez ítems con formato de respuesta tipo Likert, que debían responderse con relación al barrio actual. Por ejemplo: "Participa de alguna forma en su junta de vecinos". Se realizó un análisis factorial exploratorio que corrobora la dimensionalidad de la escala original. La confiabilidad de la escala medida mediante el coeficiente Alpha de Cronbach es de 0.909. El puntaje individual en la escala se obtuvo calculando el promedio de los diez ítems.

\section{Procedimiento}

Estudiantes de psicología capacitados encuestaron a los participantes en su residencia en septiembre de 2013. Previa aplicación del cuestionario, se explicaron los objetivos y alcances de la investigación. Se solicitó firmar un consentimiento informado, revisado y aprobado por el comité de ética de la Facultad de Medicina de la Universidad de Valparaíso.

\section{Análisis}

Se realizó un análisis descriptivo de población e instrumentos utilizados. Para probar las hipótesis de estudio se realizó un análisis de comparación de medias de las sub-escalas por sector mediante Prueba T Student. Se establecieron relaciones lineales entra las sub-escalas por sector con el coeficiente de correlación de Pearson. Se empleó el software SPSS Statistics 17.0.2.

\section{Resultados}

En la Tabla 1 se reportan las medias que consideran la muestra de Chaitén. La más elevada corresponde a PIBp (4.92), mientras que la más baja es PPC (1.57). De las variables que dan cuenta de la diferencia de tiempo, las que miden pasado (PIBp, PASBp y PAE$\mathrm{Bp}$ ) tienen todas una media mayor que las de barrio actual (PIBa, PASBa y PAEBa). En las variables de barrio pasado, la media más alta es 4.92 (PIBp), la más baja es 4.59 (PAEBp). En las medias de barrio actual, la media más alta es 2.94 (PASBa), mientras que la más baja es 2.59(PAEBa). La variable sentido de comunidad (PSCB) tiene una media de 3.61.

TABLA1.

Media obtenida para los diferentes factores en Chaitén y Constitución*

\begin{tabular}{lcccc}
\hline \multicolumn{1}{c}{ Sub-escala } & \multicolumn{2}{c}{ Sector } \\
\hline & $\mu$ & Chaitén & & \multicolumn{2}{c}{ Constitución } \\
\hline Identidad p. & 4.92 & Error típ. & $\mu$ & Error típ. \\
Identidad a. & 2.92 & 0.099 & 4.29 & 0.166 \\
Apego Social p. & 4.85 & 0.110 & 4.67 & 0.164 \\
Apego Social a. & 2.94 & 0.112 & 3.99 & 0.184 \\
Apego Espacial p. & 4.59 & 0.119 & 4.43 & 0.159 \\
Apego Espacial a. & 2.59 & 0.105 & 2.47 & 0.174 \\
Sentido Comunidad & 3.61 & 0.105 & 4.96 & 0.131 \\
Participación Cívica & 1.57 & 0.101 & 5.04 & 0.115 \\
\hline
\end{tabular}

* Todas las variables hacen referencia a la relación con el barrio pasado (p.) o actual (a.). Fuente: elaboración propia 
También en la Tabla 1 se reportan las medias que consideran la muestra de Constitución. La media más elevada corresponde a PSCB (5.04) mientras que la más baja es PAEBp (2.47). De las variables que dan cuenta de la diferencia de tiempo, las que miden barrio actual (PIBa, PASBa y PAEBa) tienen todas una media mayor que las variables de barrio pasado (PIBp, PASBp y PAEBp). En las variables de barrio pasado, la más alta es 4.29 (PIBp), mientras que la más baja es 2.47 (PAEBp). En las medias de barrio actual la media más alta es 4.96 (PAEBa) y la más baja es 4.43 (PASBa). La variable sentido de comunidad (PSCB) tiene una media de 5.04.

Las medias indicadas para promedio identidad con el barrio pasado (PIBp) y actual (PIBa) presentan para Chaitén, tal como se señala en la Tabla 1, una diferencia entre las medias que va de 4.92 (PIBp) a 2.92 (PIBa). En tanto Constitución presenta medias que van de 4.29 (PIBp) a 4.67 (PIBa) (Tabla 1). Así, mientras que en Chaitén la media desciende de PIBp a PIBa, en Constitución aumenta de PIBp a PIBa, tal como se aprecia en la Figura 1.

El promedio de apego social en el barrio pasado (PASBp) y actual (PASBa) presenta la misma si- tuación que (PIBp) y (PIBa): la direccionalidad es diferente en las medidas obtenidas para Chaitén y Constitución. Mientras que, como indica la Tabla 1, en Chaitén PASBp (4.85) es mayor que PASBa (0.94), en Constitución son inversos (PASBp $=$ 3.99; $\mathrm{PASBa}=4.43)$, siendo la dirección del cambio ascendente, como se aprecia en la Figura 2.

Las medias de apego espacial en el barrio pasado (PAEBp) y actual (PASBa) para Chaitén y Constitución varían en cuanto a la dirección entre medias menores y mayores, situación que al representarse gráficamente (Figura 3), señala en Chaitén una baja de PAEBp (4.59) a PAEBa (2.59) (Tabla 1). En tanto Constitución presenta una dirección ascendente con medias que van de PAEBp (2.47) a PAEBa (4.96) (Tabla 1).

En la Tabla 2 se presentan las diferencias de medias entre Chaitén y Constitución de todas las variables medidas. Se presentan diferencias significativas en todas ellas.

Las correlaciones de las variables medidas en la localidad de Chaitén se presentan en la Tabla 3. sentido de comunidad en el barrio (PSCB) tiene una relación significativa $(\mathrm{p}<0.01)$ con PIBa

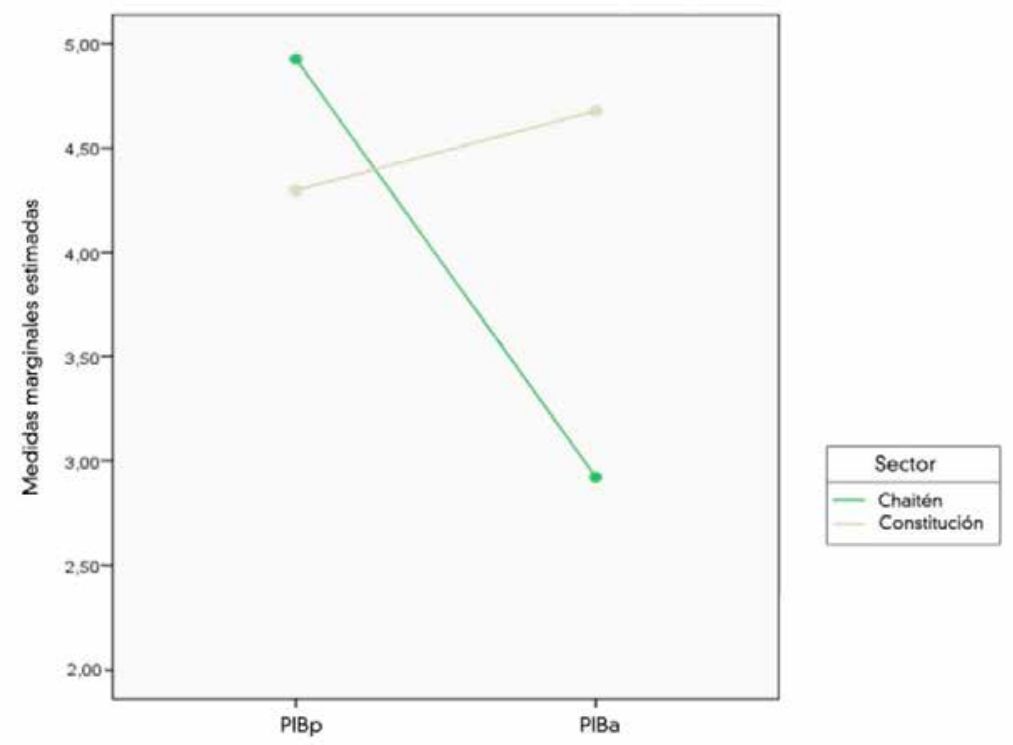

Figura 1. Media de identidad en cuanto a barrio pasado (PIBp) y actual (PIBa)

Fuente: elaboración propia 

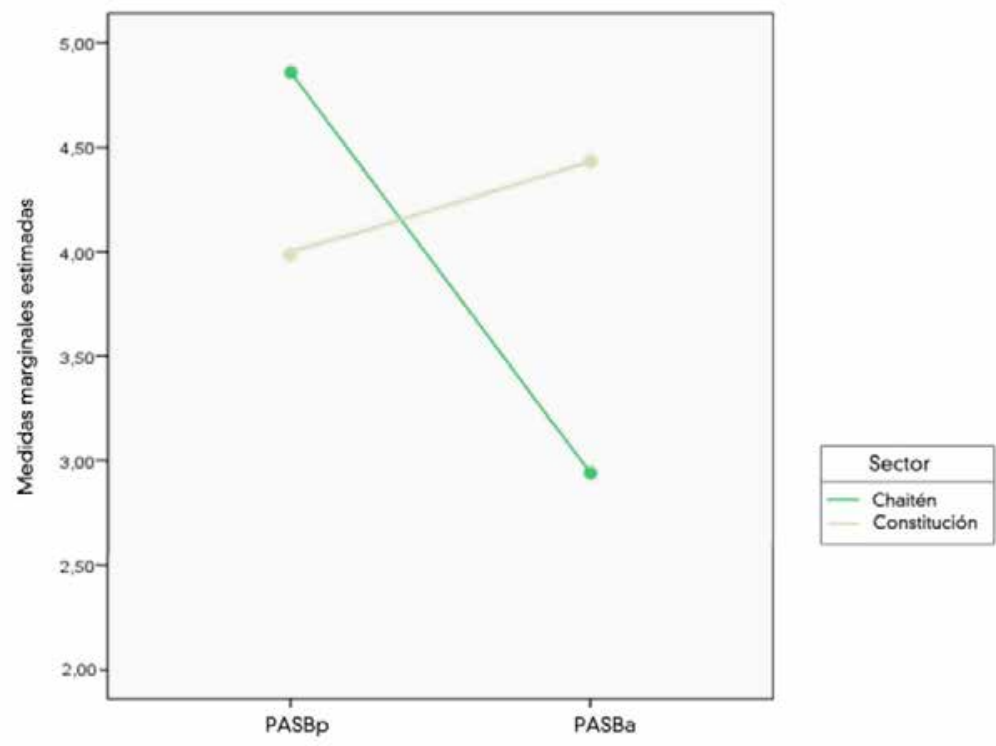

Figura 2. Media de apego social en el barrio pasado (PASBp) y actual (PASBa)

Fuente: elaboración propia

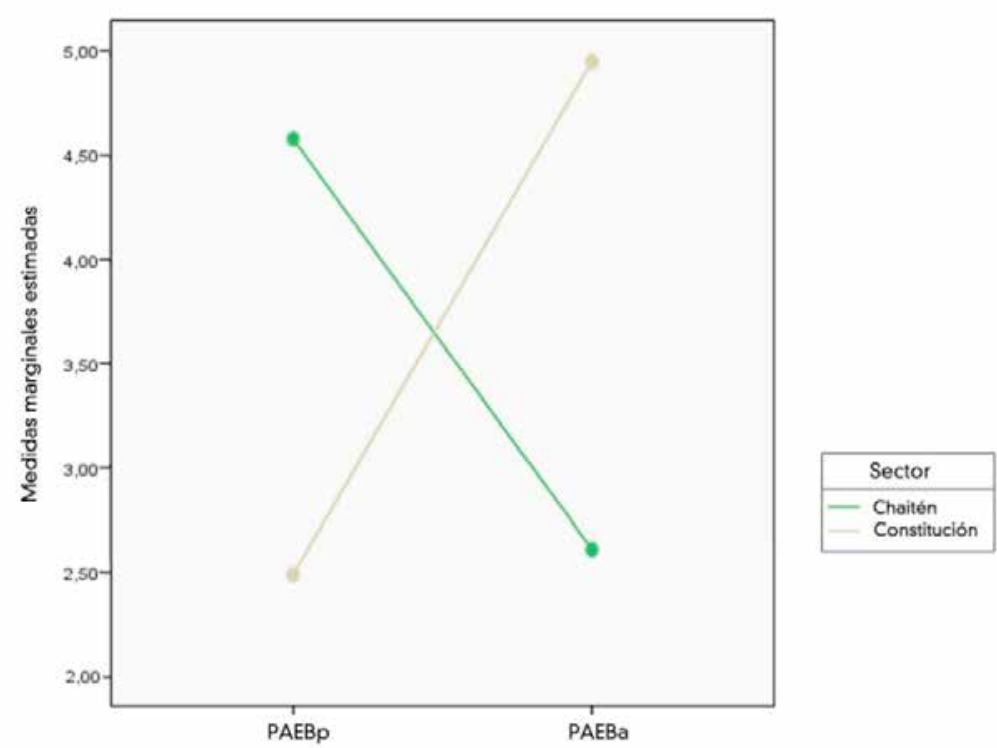

Figura 3. Media de apego espacial en el barrio pasado (PAEBp) y actual (PAEBa)

Fuente: elaboración propia 
TABLA 2.

Diferencias de media entre las muestras de Chaitén y Constitución por cada sub-escala*

\begin{tabular}{lccccccc}
\hline \multicolumn{7}{c}{ Sub-escala } & \multicolumn{7}{c}{ Diferencias relacionadas } & $\mathrm{t}$ & $\mathrm{gl}$ & Sig. (bilateral \\
\hline & Media & $\begin{array}{c}\text { Error típ. de } \\
\text { la diferencia }\end{array}$ & $\begin{array}{c}\text { 95\% Intervalo de confianza } \\
\text { para la diferencia }\end{array}$ \\
& \multicolumn{7}{c}{$\begin{array}{c}\text { Inferior } \\
\text { Superior }\end{array}$} \\
\hline Identidad p. & 0.62 & 0.18 & 0.26 & 0.98 & 3.44 & 222 & 0 \\
Identidad a. & -1.75 & 0.19 & -2.14 & -1.36 & -8.85 & 149.18 & 0 \\
Apego social p. & 0.86 & 0.20 & 0.45 & 1.26 & 4.22 & 222 & 0 \\
Apego social a. & -1.48 & 0.19 & -1.88 & -1.09 & -7.46 & 164.32 & 0 \\
Apego espacial p. & 2.11 & 0.20 & 1.71 & 2.51 & 10.38 & 137.58 & 0 \\
Apego espacial a. & -2.37 & 0.16 & -2.7 & -2.03 & -14.08 & 173.21 & 0 \\
Sentido de comunidad & -1.43 & 0.16 & -1.74 & -1.11 & -8.9 & 222 & 0 \\
Participación cívica & -0.95 & 0.21 & -1.38 & -0.52 & -4.36 & 222 & 0 \\
\hline
\end{tabular}

* Todas las variables hacen referencia a la relación con el barrio pasado (p.) o actual (a.).

Fuente: elaboración propia

TABLA 3.

Correlaciones entre Sub-escalas de Chaitén***

\begin{tabular}{lcccccccc}
\hline Correlaciones & PIBp & PIBa & PASBp & PASBa & PAEBp & PAEBa & PSCB & PPC \\
\hline Identidad p. & - & & & & & & & \\
Identidad a. & $-0.174^{*}$ & - & & & & & & \\
Apego social p. & $0.725^{* *}$ & -0.023 & - & & & & & \\
Apego social a. & -0.101 & $0.77^{* *}$ & 0.055 & - & & & & \\
Apego espacial P. & $0.72^{* *}$ & -0.08 & $0.746^{* *}$ & 0.028 & - & & & \\
Apego espacial a. & $-0.208^{*}$ & $0.637^{* *}$ & -0.032 & $0.596^{* *}$ & -0.063 & - & & \\
Sentido de comunidad & 0.083 & $0.475^{* *}$ & 0.16 & $0.587^{* *}$ & 0.146 & $0.417^{* *}$ & - & \\
Participación cívia & 0.042 & $0.305^{* *}$ & 0.139 & $0.26^{* *}$ & 0.12 & $0.348^{* *}$ & $0.506^{* *}$ & - \\
\hline
\end{tabular}

$* p<0.05$;

$* * p<0.01$

*** Todas las variables hacen referencia a la relación con el barrio pasado (p.) o actual (a.).

Fuente: elaboración propia

$(\mathrm{r}=0.47) ;$ PASBa $(\mathrm{r}=0.58)$; PAEBa $(\mathrm{r}=0.41) \mathrm{y}$ PPC $(r=0.50)$. Identidad de barrio pasado (PIBp) se relaciona de forma significativa $(\mathrm{p}<0.01)$ con PASBp $(r=0.72)$; PAEBp $(r=0.72)$; y una relación inversa significativa $(\mathrm{p}<0.05)$ débil con PIBa $(\mathrm{r}=-0.17)$ y PAEBa $(\mathrm{r}=-0.20)$. Apego social con el barrio pasado (PASBp) se relaciona significativamente $(\mathrm{p}<0.01)$ con PAEBp $(\mathrm{r}=0.76)$. Apego espacial con el barrio actual (PAEBa) tiene una relación significativa $(\mathrm{p}<0.01)$ con $\mathrm{PIBa}(\mathrm{r}=0.63)$; PASBa $(r=0.59)$. Apego social con el barrio actual (PASBa) tiene una relación significativa $(\mathrm{p}<0.01)$ con PIBa $(\mathrm{r}=0.77) ;$ PAEBa $(\mathrm{r}=0.59) ;$ y PPC $(\mathrm{p}<0.01, \mathrm{r}=0.26)$.

La Tabla 4 presenta las correlaciones de variables medidas en Constitución. Sentido de comunidad en el barrio (PSCB) correlaciona significativamente $(\mathrm{p}<0.01)$ conPIBp $(\mathrm{r}=0.40)$; PIBa $(\mathrm{r}=0.68)$; PASBp $(\mathrm{r}=0.50)$; PASBa $(\mathrm{r}=0.72)$; PAEBp $(r=0.40)$; y PAEBa $(r=0.66)$. Identidad con el barrio pasado (PIBp) correlaciona significativamente $(\mathrm{p}<0.01)$ con PIBa $(\mathrm{r}=0.45)$; PASBp $(\mathrm{r}=0.74)$; PASBa $(\mathrm{r}=0.47)$; y PAEBp $(\mathrm{r}=0.54)$. Identidad con el barrio actual (PIBa) correlaciona 
Tabla 4. Correlaciones entre sub-escalas de Constitución***

\begin{tabular}{lcccccccc}
\hline \multicolumn{1}{c}{ Correlaciones } & PIBp & PIBa & PASBp & PASBa & PAEBp & PAEBa & PSCB & PPC \\
\hline Identidad p. & - & & & & & & & \\
Identidad a. & $0.450^{* *}$ & - & & & & & & \\
Apego social p. & $0.740^{* *}$ & $0.456^{* *}$ & - & & & & & \\
Apego social a. & $0.470^{* *}$ & $0.765^{* *}$ & $0.582^{* *}$ & - & & & & \\
Apego espacial p. & $0.544^{* *}$ & $0.364^{* *}$ & $0.505^{* *}$ & $0.381^{* *}$ & - & & & \\
Apego espacial a. & $0.273^{*}$ & $0.640^{* *}$ & $0.315^{* *}$ & $0.509^{* *}$ & $0.354^{* *}$ & - & & \\
Sentido de comunidad & $0.401^{* *}$ & $0.689^{* *}$ & $0.509^{* *}$ & $0.726^{* *}$ & $0.400^{* *}$ & $0.663 * *$ & - & \\
Participación cívica & -0.01 & 0.189 & 0.013 & 0.053 & 00.212 & $0.313^{* *}$ & 0.155 & - \\
\hline
\end{tabular}

$* p<0.05 ; * * p<0.01 * * *$ Todas las variables hacen referencia a la relación con el barrio pasado (p.) o actual (a.).

Fuente: elaboración propia

significativamente con PASBp $(r=0.45)$; PASBa $(\mathrm{r}=0.76)$; PAEBa $(\mathrm{r}=0.50)$; y PSCB $(\mathrm{r}=0.68)$. Apego social con el barrio pasado (PASBp) correlaciona significativamente $(\mathrm{p}<0.01)$ conPIBa $(\mathrm{r}=$ 0.45); PASBa $(\mathrm{r}=0.58)$; PAEBp $(\mathrm{r}=0.50)$; y de forma significativa débil con PAEBa $(\mathrm{p}<0.01, \mathrm{r}=$ 0.31). Apego Espacial Barrio pasado (PAEBp) correlaciona significativamente con PASBa $(r=0.38)$.

Los resultados muestran que las personas que se mantuvieron en su lugar de origen presentan diferentes grados de apego de lugar, identidad de lugar, sentido de comunidad y participación cívica que quienes fueron desplazados.

\section{Discusión}

Los resultados de esta investigación exploratoria revelan que las dimensiones afectivas (apego de lugar) y cognitivas (identidad de lugar) con el lugar, así como las dimensiones relacionales (sentido de comunidad) y conductuales (participación) con la vecindad, son mayores para quienes se quedaron en su lugar de origen tras la ocurrencia del desastre (Constitución).

En Chaitén para la comunidad desplazada y disgregada a consecuencia de una erupción volcánica los vínculos espaciales (apego de lugar e identidad de lugar) con el barrio donde actualmente residen son diferentes a los reportados con su barrio de origen (Tabla 1). Los valores de las medias para apego de lugar e identidad de lugar no superan los 3 puntos, mientras que las medias relacionadas con el barrio anterior son todas superiores a 4.5. La mayor diferencia se produce entre la identidad de lugar con el barrio actual (2.92) y el barrio anterior (4.92), lo que apoya los planteamientos de la disrupción de los vínculos espaciales y sus manifestaciones paradójicas. Estos resultados son consistentes con los hallazgos de Ruiz y Hernández (2014), quienes tras estudiar los vínculos antes-después con el lugar en una comunidad afectada por la erupción de un volcán en las Islas Canarias en España, explican que las diferencias a favor del lugar pasado se deben a que el apego de lugar se construye a partir de una experiencia de continuidad, fruto de las interacciones cotidianas con el espacio, que usualmente se mantienen en el tiempo aún cuando las personas son forzadas a abandonarlo. La identidad de lugar, por ser parte de la identidad social, es un vínculo más estable y por tanto más difícil de modificar.

En Constitución, cuyas residencias fueron reconstruidas en el mismo lugar tras ser destruidas a consecuencia de un terremoto, las medias de los vínculos espaciales (apego espacial e identidad de lugar) con el barrio donde actualmente residen son mayores a 4.6, mientras que las medias en relación al barrio anterior no superan 4.3. En este caso, la mayor diferencia se produce entre el apego espacial con el barrio actual (4.96) y el barrio anterior (2.47). Estos resultados pueden asociarse a los estudios que plantean que el apego de lugar es importante para el grado de resiliencia que alcanzan las comunidades enfrentadas a desastres (Bird et al., 2011; Norris et al., 2008). 
Las medias para las dimensiones relacionales con la vecindad, en el caso de Chaitén, muestran un valor medio de 3.61 para sentido de comunidad y muy bajo (1.57) para participación cívica. En cambio, en Constitución muestran un valor muy alto (5.04) para sentido de comunidad y bajo $(2,53)$ para participación cívica. Si consideramos que en el caso de Chaitén la comunidad fue desmembrada tras la catástrofe, mientras que en Constitución la comunidad se mantuvo, los resultados confirman dos aspectos centrales de la relación entre los vínculos relacionales y la noción de comunidad: que una ruptura en la red de relaciones de dependencia mutua (Sarason, 1974) impactan profundamente el sentido de comunidad y segundo, que como plantea Gilchrist (2009), estas redes son la base del compromiso colectivo que permiten el efectivo desarrollo comunitario. Por tanto, mantener los vínculos comunitarios es un factor central a considerar en toda estrategia de desplazamiento o reconstrucción tras una catástrofe.

En esta misma línea y como lo muestran los gráficos en las figuras 1, 2 y 3, la comparación de medias entre ambos barrios para las variables de vínculos espaciales antes y después, muestran una direccionalidad inversa. En el caso de Chaitén todos los valores presentan diferencias significativas a favor del barrio anterior, en cambio en Constitución la relación es a favor del barrio actual. Estos resultados confirman la extensa evidencia del impacto negativo del desplazamiento en las comunidades (Fried, 1963; Gibson, 2007), en especial en las comunidades pobres (Manzo, 2014).

Los resultados nos muestran que el desplazamiento forzado por una catástrofe es una experiencia emocional negativa que puede contribuir al fortalecimiento del apego con el lugar que se abandona. Constatación que es consistente con lo que un grupo de autores denominan "el lado oscuro del apego al lugar" (Chawla, 1992), metáfora utilizada para cuestionar el excesivo énfasis que la literatura atribuye a los afectos y experiencias positivas en la conformación del apego hacia lugares residenciales. Desde esta posición crítica, se relevan los efectos de sentimientos y experiencias negativas, sugiriendo que el apego de lugar puede involucrar una tensión dinámica entre la pertenencia, la exclusión y los afectos positivos y negativos (Manzo, 2014).

Finalmente respecto a la asociación entre las variables estudiadas, se encontraron para Chaitén correlaciones significativas en relación a su barrio actual por sobre $\mathrm{r}=0.4$ entre apego de lugar, identidad de lugar y sentido de comunidad. Mientras que en Constitución la asociación entre estas variables fue tanto para el barrio pasado como para el barrio actual.

La identidad de lugar y el apego de lugar en ambas localidades correlacionan significativamente con relación al barrio anterior y al actual por sobre $\mathrm{r}=$ 0.4. Estos resultados confirman la extensa evidencia de la estrecha relación entre ambos constructos (Brown \& Werner, 1985; Hernández et al., 2007; Stedman, 2002).

\section{Conclusiones}

Hemos constatado cómo las transformaciones producidas a partir de los desastres y los subsecuentes procesos de reconstrucción y/o desplazamiento alteran los entornos físicos de las personas, modificando la construcción del simbolismo y la significación espacial, lo cual afecta las dinámicas de convivencia y asociación de las comunidades. Como plantean Días y Prewitt Dayal (2008), el sentimiento de pérdida de lugar es el impacto más catastrófico de un desastre, razón por la cual los procesos de reconstrucción post-desastre deben, considerar no solo la reposición de las estructuras físicas sino también, tal como plantean Rosales y Salazar (2010), afrontar aspectos sociales y psicológicos. Por tanto la inclusión del conocimiento de los vínculos socioespaciales de las personas que han sido desplazadas a consecuencia de iniciativas post desastres, puede mejorar los procesos de reconstrucción.

Los resultados de este trabajo suponen un primer paso en la exploración del apego y la identidad de lugar, el sentido de comunidad y la participación cívica en personas afectadas por catástrofes socionaturales. Para futuras investigaciones sería interesante explorar otras variables que en han mostrado su efecto en varios estudios, como el régimen de tenencia de la vivienda (Riger \& Lavrakas, 1981) y el 
número de viviendas anteriores (Cuba \& Hummon, 1993), que pretendo presentar en futuros avances de esta investigación.

Otra limitación de los resultados es la simplificación de las relaciones conceptuales propuestas debido al enfoque metodológico adoptado. Algunas alternativas para abordar dicha limitación podrían ser la replicación con otras muestras y la complementariedad con estudios longitudinales. Otro aspecto previsto en esta investigación, como se apunta en la introducción, es triangular el abordaje metodológico con un análisis cualitativo para explorar desde la experiencia, la participación en el barrio de los participantes y los factores vinculados al apego e identidad de lugar, además del sentido de comunidad, en el contexto de la reconstrucción post-desastre.

\section{Referencias}

Banyard, V., \& Williams, L. (2007). Women's voices on recovery: A multi-method study of the complexity of recovery from child sexual abuse. Child Abuse ES Neglect, 31(3), 275-290.

Bird, D., Gísladóttir, G., \& Dominey-Howes, D. (2011). Different communities, different perspectives: Issues affecting residents' response to a volcanic eruption in southern Iceland. Bull Volcanol, 73, 1209-1227.

Bonaiuto, M., \& Bonnes, M. (2000). Social-psychological approaches in environment-behaviour studies. Identity Theory and the Discursive Approach. En S. Wapner, J. Demick, T. Yamamoto, \& H. Minami (Eds.). Theoretical perspectives in environment-behaviour research (67-78). New York: Kluwer Academic / Plenum Publishers.

Bonanno, A., Galea, S., Bucciarelli, A., \& Vlahow, D. (2006). Psychological resilience after disaster. Psychology science, 17(3), 181-186.

Brown, B., \& Perkins, D. (1992). Disruptions in place attachment. In I. Altman and S. Low (Eds.), Place Attachment (pp. 279-304). New York: Plenum Press.

Brown, B., \& Werner, C. (1985). Social cohesiveness, territoriality, and holiday decorations: The influence of cul-de-sacs. Environment and Behavior, 17(5), 539-565.
Chawla, L. (1992). Childhood place attachments. In I. Altman \& S. Low (Eds.), Place Attachment (pp. 63-84). New York: Plenum Press.

Cuba, L., \& Hummon, D. (1993). A place to call home: identification with dwelling, community, region. The Sociological Quarterly, 34, 111-131.

Fried, M. (1963). Grieving for a lost home. In L. Duhl (Ed.), The urban condition (pp. 151-171). New York: Basic Books.

García, F., Reyes, A. \& Cova, F. (2014). Severidad del trauma, optimismo, crecimiento postraumático y bienestar en sobrevivientes de un desastre natural. Universitas Psychologica, 13(2), 575-584.

Gibson, K. (2007). Relocation of the Columbia Villa Community: views from residents. Journal of Planning Education and Research, 27, 5-19.

Gilchrist, A. (2009). The well connected community: networking to the edge of chaos. Bristol England: Policy Press.

Greenfield, E., \& Marks, N. (2010). Sense of community as a protective factor against long-term psychological effects of childhood violence. Social Service Review, 84(1), 129-147.

Heller, T. (1982). The Effects of involuntary residential relocation: A review. American Journal of Community Psychology, 10, 471-492.

Hernández, B., Hidalgo, M., Salazar-Laplace, M., \& Hess, S. (2007). Place attachment and place identity in natives and non-natives. Journal of Environmental Psychology, 27(4), 310-319.

Hidalgo, M., \& Hernández, B. (2001). Place attachment: Conceptual and empirical questions. Journal of Environmental Psychology, 21(3), 273-281.

Ibieta, G., (2013). Informe socio histórico de Constitución. Documento no publicado, realizado en el marco del proyecto FONDECYT 11121596 "Vínculos socio-espaciales en contextos de transformación urbana producida por catástrofes naturales". Universidad de Valparaíso, Chile.

Joh, H. (1997). Disaster stress of the 1995 Kobe earthquake. Psychologia: An International Journal of Psychology in the Orient, 40, 192-200.

Korosec-Serfaty, P. (1976). Appropriation of space. Proceedings of the Strasbourg conference. IAPC-3. Strasbourg-Lovaine La Neuve: CIACO. 
Larrañaga, O., \& Herrera, R. (2010). Encuesta post terremoto: Principales resultados efectos en la calidad de vida de la población afectada por el terremoto/ tsunami. Chile: Ministerio de Planificación.

Lewicka, M. (2011). Place attachment: How far have we come in the last 40 years? Journal of Environmental Psychology, 31(3), 207-230.

Long, D., \& Perkins, D. (2003). Confirmatory factor analysis of the sense of community index and development of a brief SCI. Journal of Community Psychology, 3, 279-296.

Manzo, L. (2014). The Shadow Side of Place Attachment. In L. Manzo \& P. Devine-Wright (Eds.), Place Attachment. Advances in Theory, methods and applications (pp. 178-190). London: Routledge.

Manzo, L., \& Perkins, D. (2006). Finding common ground: The importance of place attachment to community participation and planning. Journal of Planning Literature, 20(4), 335-350.

Marchant, J. (2010). Lágrimas de ceniza, Estudio cualitativo sobre la experiencia de desplazamiento de los habitantes de Chaitén, asentados en las ciudades de la Isla de Chiloéy Puerto Montt. Chile: Oficina Nacional de Emergencia, Gobierno de Chile.

Mihaylov, N., \& Perkins, D. (2014). Community Place Attachment and its Role in Social Capital Development. In L. Manzo \& P. Devine-Wright (Eds.), Place Attachment. Advances in Theory, methods and applications (pp. 61-73). London: Routledge.

Norris, F., Friedman, M., \& Watson, P. (2002). 60.000 disaster victims speak: part II. Summary and implications of the disaster mental health research. Psychiatry: Interpersonal and biological processes, 65, 240-260.

Norris, F., Stevens, S. Pfefferbaum, B., Wyche, K., \& Pfefferbaum, R. (2008). Community resilience as a metaphor, theory, set of capacities, and strategy for disaster readiness. American Journal of Community Psychology, 41, 127-150.

Norris, F., Watson, P., Hamblen, J., \& Pfefferbaum, B. (2005). Provider perspectives on disaster mental health services in Oklahoma City. Journal of Aggression, Maltreatment, and Trauma, 10, 649-661.

Nowel, B., \& Boyd, N. (2010). Viewing community as responsibility as well as resource: deconstructing the theoretical roots of psychological sense of community. Journal of Community Psychology, 38(7), 828-841.

Perkins, D. D., Florin, P., Rich, R. C., Wandersman, A., \& Chavis, D. M. (1990). Participation and the social and physical environment of residential blocks: Crime and community context. American Journal of Community Psychology, 18(1), 83-115.

Perkins, D., \& Long, D. (2002). Neighborhood sense of community and social capital: A multi-level analysis. In A. T. Fisher, C. C. Sonn \& B. J. Bishop (Eds.), Psychological sense of community: Research, applications, and implications. (pp. 291-318). New York, NY, US: KluwerAcademic/PlenumPublishers.

Pol, E. (2002). El modelo dual de la apropiación del espacio. En R. García Mira, J. M. Sabucedo \& J. Romay (Eds.), Psicología y Medio Ambiente. Aspectos psicosociales, educativos y metodológicos (pp. 123-132). A Coruña: Asociación galega de estudios e investigación psicosocial.

Prewitt Diaz, J. O., \& Dayal, A. (2008). Sense of place: A model for community based psychosocial support programs. Australasian Journal of Disaster and Trauma Studies, 63(8), 820-827.

Proshansky, H., Fabian, A., \& Kaminoff, R. (1983). Place-identity: Physical world socialization of the self. Journal of Environmental Psychology, 3(1), 57 83.

Razeto, A. (2013). Potenciando el desarrollo local de comunidades afectadas por desastres. Revista INVI, 28(77), 111-136.

Riger, S. \& Lavrakas, P. (1981). Community ties: Patterns of attachment and social interaction in urban neighborhood. American Journal of Comunity Psychology, 9, 55-66.

Rosales, V. \& Salazar, F. (2010). Los procesos de reconstrucción: reto para la sociedad y para los gobiernos. Costa Rica: Comisión Nacional de Emergencias. Recuperado de http://www.cne.go.cr/Documentos/ mitigacion/el caso cinchona.pdf

Ross, C. T., Bonaiuto, M., \& Breakwell, G. M. (2003). Identity theories and environmental psychology. Psychological Theories for Environmental Issues. (pp. 203-234). Aldershot: Ashgate

Ruiz, C., \& Hernández, B. (2014). Emotions and coping strategies during an episode of volcanic activity 
and their relations to place attachment. Journal of Environmental Psychology, 38, 279-287.

Sanders, S., Browie, S., \& Bowie, Y. (2003). Lessons learned on forced relocation of older adults: the impact of hurricane andrea on health, mental health, and social support of public housing residents. Journal of Gerontological Social Work, 40, 23-35.

San Martín, R. (2010). Informe final en investigación especial de la intendencia regional de Los Lagos. Los Lagos: Contraloría general de la República. Contraloría regional de Los Lagos.

Sarason, S. (1974). The psychological sense of community: Prospects for a Community Psychology. San Francisco: Jossey-Bass.

Scannell, L., \& Gifford, R. (2010a). Defining place attachment: A tripartite organizing framework. Journal of Environmental Psychology, 30(1), 1-10.

Scannell, L., \& Gifford, R. (2010b). The relations between natural and civic place attachment and pro environmental behavior. Journal of Environmental Psychology, 30(3), 289-297.
Stedman, R. C. (2002). Toward a social psychology of place predicting behavior from place-based cognitions, attitude, and identity. Enviroment and behavior, 34(5), 561-581.

Twigger-Ross, C. L., Bonaiuto, M., \& Breakwell, G. (2003). Identity theories and environmental psychology. En M. Bonnes, T. Lee \& M. Bonaiuto (Eds.), Psychological theories for environmental issues (pp. 203-233). England: Ashgate Publishing Limited.

Vidal, T., Berroeta, H., Di Masso, A., Valera, S., \& Peró, M. (2013). Apego al lugar, identidad de lugar, sentido de comunidad y participación en un contexto de renovación urbana. Estudios de Psicología, 34 (3), 275-286.

Vidal, T., \& Pol, E. (2005). La apropiación del espacio: Una propuesta teórica para comprender la vinculación entre las personas y los lugares. Anuario de Psicología, 36(3), 281-297.

Vidal, T., Valera, S., \& Peró, M. (2010). Apego al lugar, identidad de lugar y movilidad residencial en estudiantes de grado. Psyecology, 1(3), 291-307. 
